\title{
positron emission tomography in neuroscience research
}

Positron Emission Tomography (PET) is useful for experimental models of motor neuron disorder, Parkinson's disease, Alzheimer's disease, Stroke, Epilepsy' etc. It enables the acquisition of images of brain's activity by injecting short-lived radioactive chemicals into the experimental animals and by capturing the gamma rays emitted by markers. Fluorodeoxy glucose, which is an analog of glucose, is extensively used in analyzing the metabolic rate of neurons, by mapping the regions of brain involved during behavioral tasks. Alzheimer's disease and motor neuron disease are characterized by the reduction in the cerebral metabolic rate of glucose due to neuronal degeneration. ${ }^{2,3}$ With the use of PET, the regions of neuronal degeneration can be identified in real time without the need to sacrifice animals. In the Alzheimer's animal model, amyloid plaques formation in the brain of the animal can be examined using PET probe, Pittsburgh Compound-B (PIB). ${ }^{4}$ In Parkinson's animal model, [F ${ }^{18}$ ]Fluro-L-Dopa, ${ }^{5}$ in which $\mathrm{F}^{18}$ is substituted for hydrogen, can be used in studying the dopamine synthesis in the brain using transplantation experiments. Some of these experimental models have already been established in the Neuroscience Research lab at PGI, Chandigarh and are being used for drug discovery and validating biotherapeutics. The finding of PET in multiple regions of our country can greatly accelerate the advancements in the field of Neuroscience.

The regional cerebral blood flow, metabolism of glucose after infarction and oxygen consumption in the infarcted region of experimental model can also be simultaneously studied using PET which can be useful in validating therapies. ${ }^{6}$ Using FDG-PET, size of the infarct can also be measured which was previously done by histological means such as TTC. As calcium mediated neuronal damage occurs in infarcted tissue, isotope cobalt-55, a tracer of calcium has also been used for examining the infarct tissues. ${ }^{7}$ Development of new radioligands like [O-methyl-11C] $\mathrm{N}$ acetyl- $N$-(2-methoxybenzyl)-2-phenoxy5 -pyridinamine ([11C]PBR28) ${ }^{8}$ can be useful for analyzing stroke related changes and studying their rescue upon delivery of test drugs

PET can also be used in studying the protein expression levels in the brain of ani- mal models under in vivo conditions. Cai et al. have reported the levels of VEGFR in the brain of rat MCAo stroke model using PET. ${ }^{9}$ The gene expression studies can also be conducted at different time points in the brain providing real time information about molecular profile of candidate genes. ${ }^{9}$

Using FDG-PET, brain's response to the neuronal cell transplantation in the treatment of stroke has also been studied. ${ }^{10}$ PET has been used in localising of the transplanted stem cells in the mouse model of stroke. ${ }^{11}$ Development of probes for identifying specific markers in transplanted cells will enable the study of fate of the transplanted cells in in vivo boosting the national stem cell program in the country.

\section{doi : 10.5214 /ans.0972.7531.1118202}

\section{Vanitha. N}

Mammalian Genetics Unit, Centre for DNA fingerprinting and Diagnostics, Tuljaguda, Nampally, Hyderabad - 500001

\section{References}

1. Juhasz $C$ and Chugani HT. Imaging the epileptic brain with positron emission tomography. Neuroimaging Clin N Am 2003: 13(4): 705-16.

2. Fukuyama $\mathrm{H}$, Ogawa $\mathrm{M}$, Yamauchi $\mathrm{H}$, et al. Altered cerebral energy metabolism in Alzheimer's disease: A PET Study, J Nucl Med 1994; 35: 1-6.

3. Dalakas MC, Hatazawa J, Brooks RA et al. Lowered cerebral glucose utilization in amyotrophic lateral sclerosis. Ann of Neurol 1987; 22(5): 580-586.

4. Shin H, Lee YB, Kim SJ, et al. Multitracer PET imaging of amyloid plaques and neurofibrillary tangles in Alzheimer's disease. Neuroimage 2008; 43(2): 236244.

5. Bruck A, Aalto $S$, Nurmi E, et al. Cortical 6-[18F]fluoro-L-dopa uptake and fronta cognitive functions in early Parkinson's disease. Neurobiol Aging 2005; 26(6): 891-898.

6. Heiss WD and Herhoz K. Assessment of pathophysiology of stroke by positron emission tomography. Eur J of Nucl Med 1994; 21(5): 455-465.

7. Reuck DE, Santens P, Keppens J, et al. Cobalt55 positron emission tomography in recurrent ischaemic stroke. Clin Neurol Neurosurg 1999; 101(1): 15-18.

8. Kreisl WC, Mbeo G, Fujita M et al. Stroke Incidentally Identified Using Improved Positron Emission Tomography for $\mathrm{Mi}$ croglial Activation. Arch Neurol. 2009; 66(10): 1288-1289.
9. Cai W, Guzman R, Hsu AR, et al. Positron Emission Tomography Imaging of poststroke Angiogenesis. Stroke 2009; 40: 270-277.

10. Meltzer CC, Kondziolka D, Villemagne VL, et al. Serial $\left[{ }^{18} \mathrm{~F}\right]$ Fluorodeoxyglucose Positron Emission Tomography after Human Neuronal Impla ntation for Stroke. Neurosurgery 2001; 49: 586-592.

11. Sun N, Lee $A$ and Wu JC. Long term non-invasive imaging of embryonic stem cells using reporter genes. Nature Protocols 2009; 4: 1192-1201. 